Gesnerus 57 (2000) 84-87

Short Communication

\title{
Au sujet des prénoms de Tissot (1728-1797)
}

Kazimierz Karbowski

\section{Summary}

In different registers, dictionaries and publications the first names of the famous doctor Tissot are "David", "Auguste" or, absolutely wrong "SimonAndré". After the baptismal Register of the village of Grancy, his birth place, his correct first names are Samuel, Auguste, André, David or - shortened Samuel Auguste.

\section{Résumé}

Dans les différents registres, dictionnaires et publications, les prénoms du célèbre Docteur Tissot sont indiqués soit comme David, soit comme Auguste, soit - tout à fait faussement - «Simon-André». Correctement on doit l'appeler d'après les données du registre des baptêmes de la commune de Grancy, son lieu de naissance, c'est-à-dire: Samuel, Auguste, André, David, soit, en bref, Samuel Auguste.

Dans sa biographie Essai sur la vie de Tissot, Charles Eynard cite des extraits du journal intime («livre de raison») de Pierre Tissot, concernant le baptême de son fils, le futur docteur ${ }^{1}$. Né le 20 mars, il a été baptisé le 9 avril 1728: Samuel-Auguste-André-David. Eynard mentionne qu'en 1741 Tissot fut immatriculé «dans l'auditoire des belles-lettres» à Genève sous le prénom de David $^{2}$, mais en évoquant son séjour ultérieur à Montpellier il le nomme

1 Eynard, Ch., Essai sur la vie de Tissot, Lausanne 1839, pp. 10-11.

2 Eynard, Ch., op. cit., p. 14.

Prof. Dr med. Kazimierz Karbowski, Clinique Neurologique, Hôpital Universitaire, CH-3010 Berne 
«Auguste»3. On pourrait donc supposer que, dans différentes périodes de sa vie, Tissot préférait l'un de ces deux prénoms.

Tissot lui-même signait ses lettres uniquement avec son nom de famille, sans indiquer ses prénoms. C'est aussi le cas pour la majorité de ses publications. On trouve les initiales S.A.D. (Samuel Auguste David) dans la lettre du 30 janvier 1766 de «l'advoyer et conseil de la ville de Berne», contenant la nomination de Tissot comme professeur de médecine à l'Académie de Lausanne $e^{4}$, dans la publication contenant sa leçon inaugurale dans cette Académie («Sermo inauguralis de valetudine litteratorum») ${ }^{5}$, ainsi que dans l'inscription d'une plaque de marbre placée en l'honneur de Tissot en 1783 dans la cour de l'Université de Pavie, ou il a enseigné la médecine pendant deux ans ${ }^{6}$. Louis Maillet, l'auteur d'une remarquable plaquette sur les maisons anciennes de Grancy, lieu de naissance du docteur Tissot, définit les initiales des prénoms de Tissot «D.A.», c'est-à-dire «David» comme le nom principal, tout en indiquant dans une autre page de sa publication, qu'il s'appelait en réalité «Samuel-Auguste-André-David»?.

Dans les dictionnaires et lexiques les indications concernant les prénoms du Docteur Tissot diffèrent d'une façon étonnante. La Biographie Universelle Ancienne et Moderne, le nomme Simon-André8. On trouve les mêmes indications dans le Biographisches Lexikon der hervorragenden Aerzte ${ }^{9}$, ainsi que dans le Schweizer Lexikon ${ }^{10}$.

Le Dictionnaire historique et biographique de la Suisse (DHBS), appelle Tissot «Auguste»11. Le même prénom est habituellement utilisé pour lui à Lausanne. On trouve dans cette ville l'avenue Dr. Auguste Tissot ainsi qu'un auditoire "Auguste Tissot», au Centre Hospitalier Universitaire Vaudois, et les publications provenant des Institutions cantonales et universitaires indiquent «Auguste», comme prénom du docteur Tissot ${ }^{12}$.

3 Eynard, Ch., op. cit., p. 16.

4 Il s'agit d'une lettre en allemand, traduite en français dans la marge. Elle est déposée, sous le code général IS 3784, au Département des Manuscrits de la Bibliothèque cantonale et universitaire de Lausanne.

5 Tissot, S. A. D., Sermo inauguralis de valetudine litteratorum. Lausannae 1766.

6 Karbowski, K., Samuel Auguste Tissot et son "Traité de l'épilepsie» de 1770, Lausanne 1984, p. 51.

7 Maillet, L., Village de Grancy. Ses maisons anciennes et notes historiques et familles. Mauraz 1978 , pp. 2, 5 et 75.

8 Biographie Universelle Ancienne et Moderne. Tome Quarante-Sixième, Paris 1826, p. 136.

9 Hirsch, A. (éd.); Biographisches Lexikon der hervorragenden Aerzte aller Zeiten und Völker, Vol. 5, München/Berlin ${ }^{3} 1962$, p. 594.

10 Schweizer Lexikon in sieben Bänden, 7. Band, Zürich 1948, p. 383.

11 Attinger, V., Godet, M., Turler, H. (éds); Dictionnaire Historique \& Biographique de la Suisse. Tome sixième, Neuchâtel 1932, p. 618.

12 Emch-Dériaz, A.; «Auguste Tissot (1728-1797)», dans: L'Eveil médical vaudois 1750-1850, Université de Lausanne 1987, pp. 7-49. Corsini, S.; A l'ombre des Lumières. Un médecin lausannois et ses patients. Auguste Tissot 1728-1797, Lausanne 1997. 


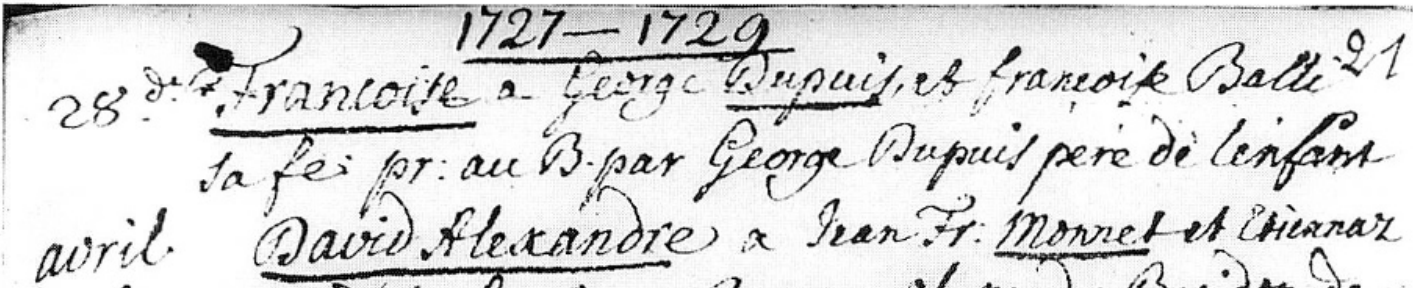
$20^{2}$. Solliard a fer pr: au $\mathrm{B}$ par. Llexandre Baridon de mentlaville, David sollias, anne Rode Tiffot of

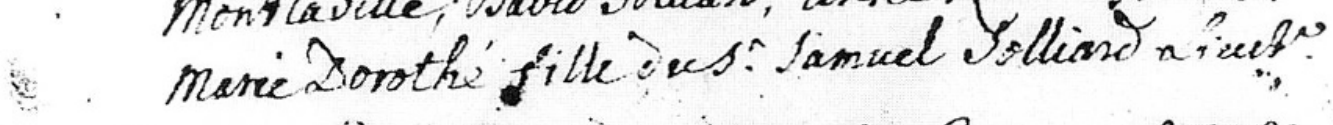

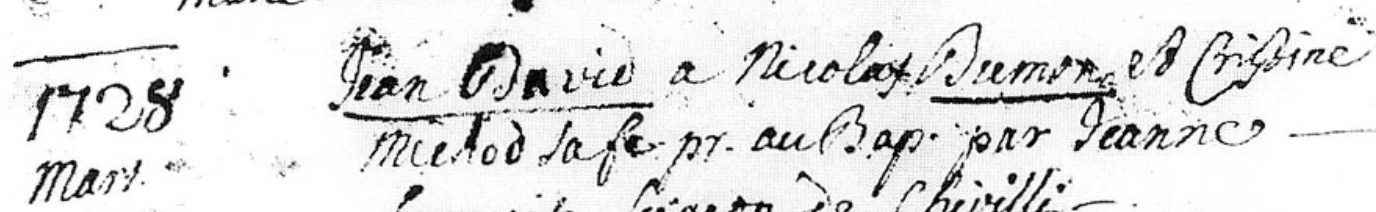

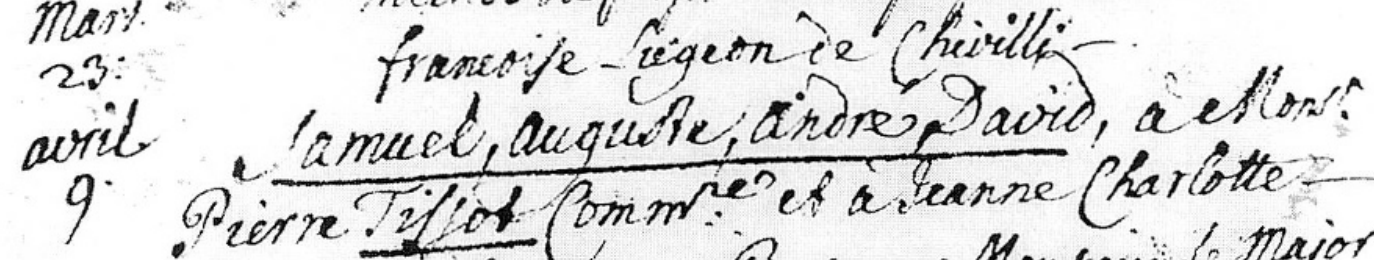

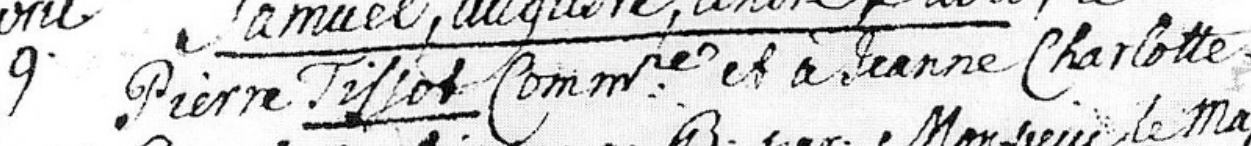

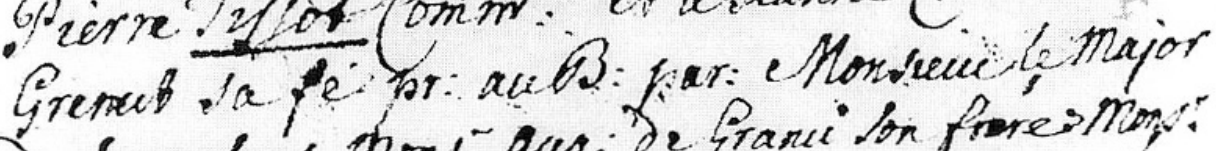

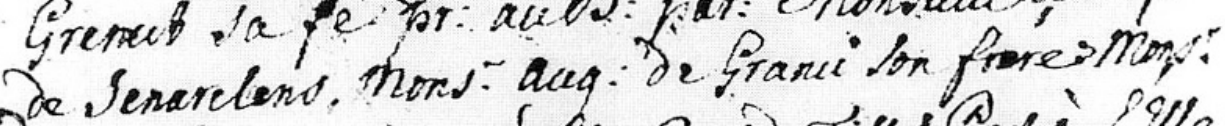

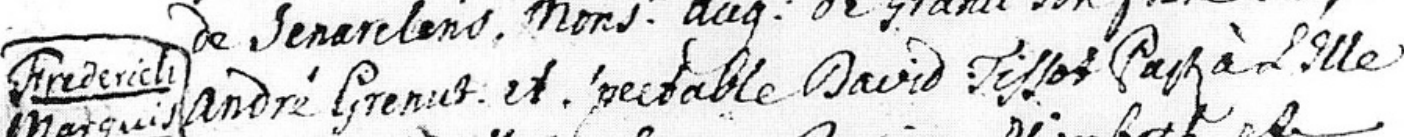

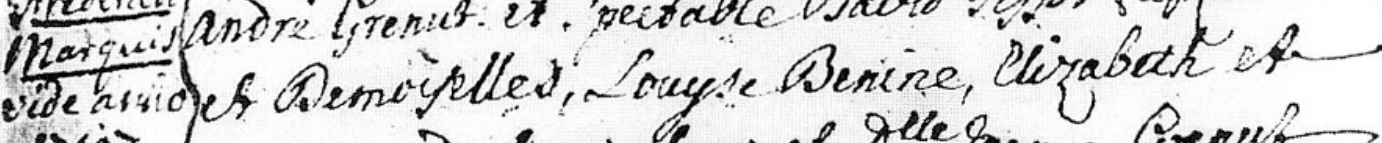
13it Srevanne de Senaritens, ef Dlle anne Grewo

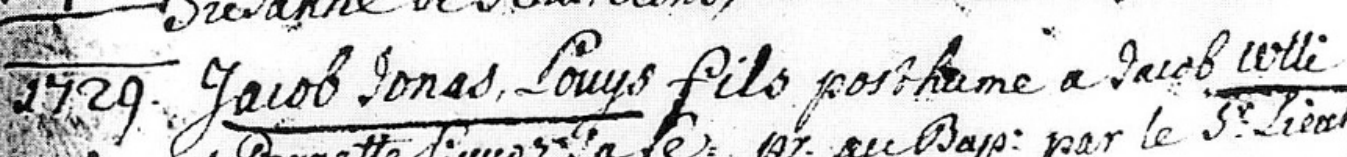

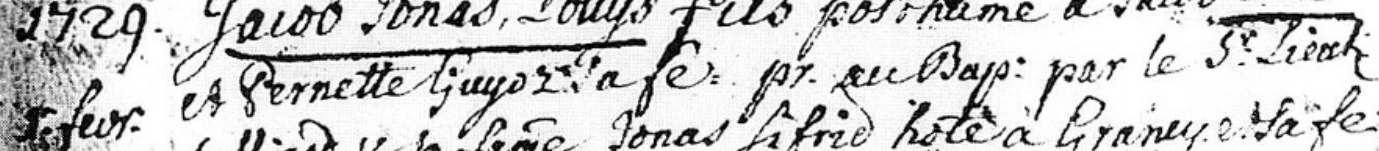

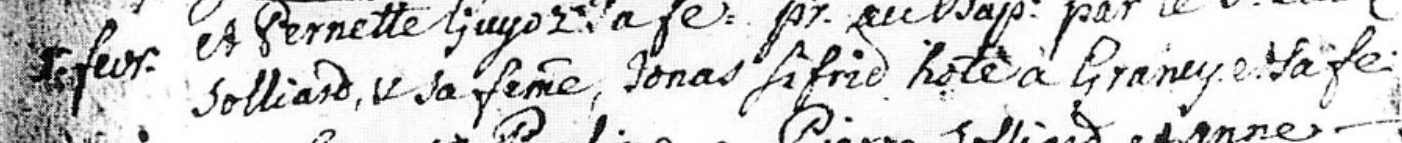

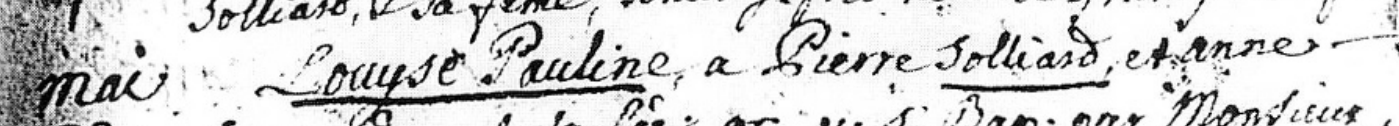

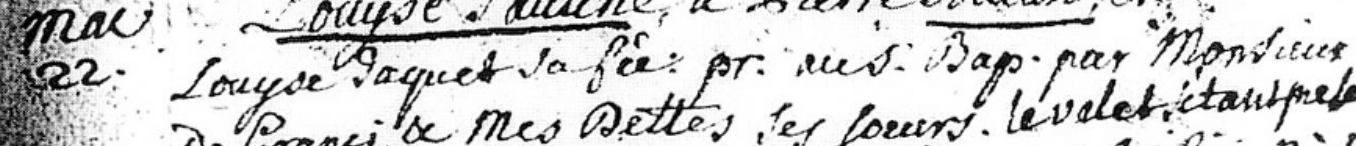

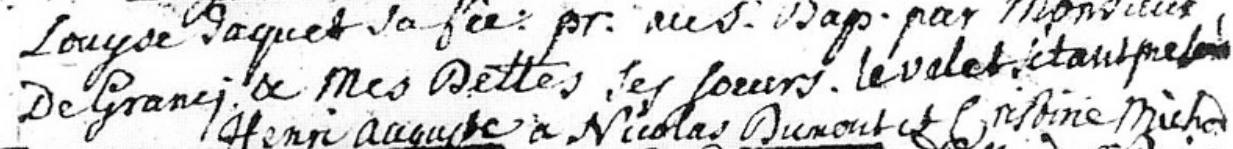

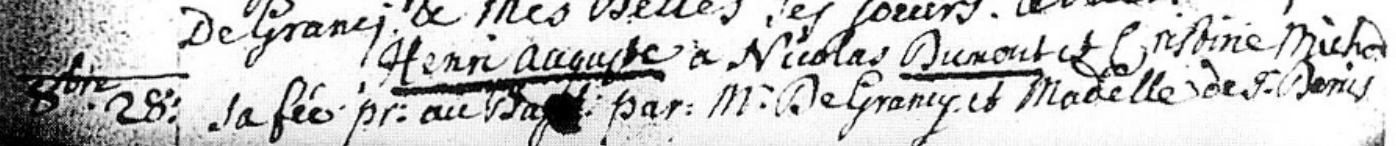

Fig.1. Extrait du registre des baptêmes de la commune de Grancy de 1712 à 1781 (ACV,Eb 63/3, p. 21) contenant les prénoms du futur docteur Tissot. Mis aimablement à disposition par la Direction des Archives cantonales vaudoises. 
Les prénoms Samuel-Auguste-André-David sont indiqués dans le Dictionnaire Biographique des Genevois et Vaudois ${ }^{13}$, ainsi que chez Eugène Olivier, dans son œuvre Médecine et Santé dans le Pays de Vaud au XVIII siècle ${ }^{14}$.

Le registre des baptêmes de la commune de Grancy contient les mêmes prénoms, toutefois sans traits d'union entre eux (fig. 1). D'après ce registre, ainsi que d'après les indications d'Eynard ${ }^{15}$, on doit admettre que Tissot porte les prénoms de ses quatre parrains. Samuel, comme le major de Senarclens, issu d'une famille de seigneurs de Grancy; Auguste d'après le frère de celuici, qui s'appelait Guillaume-Auguste; André comme André Grenus, oncle maternel de Tissot, et David comme son oncle paternel, pasteur à Lisle.

Sur la base de toutes ces données on doit conclure ce qui suit: les indications «Simon-André», contenues dans la Biographie Universelle, sont décidément fausses. Le prénom "Auguste», indiqué dans le DHBS et familier au Lausannois, semble être choisi arbitrairement parmi d'autres prénoms de Tissot. Pour prévenir les malentendus on doit l'appeler, conformément aux données du registre des baptêmes de sa commune d'origine, c'est-à-dire: Samuel, Auguste, André, David, soit, en bref, Samuel Auguste.

13 Montet de, A.; Dictionnaire Biographique des Genevois et des Vaudois, Tome second, Lausanne 1878, p. 566.

14 Olivier, E.; Médecine et Santé dans le Pays de Vaud au XVIII siècle. Tome second, Lausanne 1939, p. 1061.

15 Eynard, Ch., loco citato (1). 